\title{
JUNO Physics program
}

\section{Cécile Jollet ${ }^{a, *}$ on behalf of the JUNO collaboration}

${ }^{a}$ Univ. Bordeaux, CNRS, CENBG, UMR 5797, F-33170 Gradignan, France

E-mail: cecile.jollet@cenbg.in2p3.fr

The Jiangmen Underground Neutrino Observatory (JUNO) is a 20 kton multi-purpose liquid scintillator detector currently being built in a dedicated underground laboratory in China, expected to start data taking in 2022. JUNO's primary physics goal is the determination of the neutrino mass ordering, with an expected significance of 3-4 sigma in about six years of data taking, by measuring the oscillation pattern of electron antineutrinos coming from two nuclear power plants at a baseline of $53 \mathrm{~km}$. To reach the proposed goal an unprecedented energy resolution of $3 \%$ at $1 \mathrm{MeV}$ is needed. Besides the main physics goal, JUNO will have a very rich physics program including the measurement of neutrino oscillation parameters with a sub-percent precision, and the detection of solar, galactic core-collapse supernova and atmospheric neutrinos. JUNO will also implement a dedicated multi-messenger trigger system to maximize the potential as a neutrino telescope. In addition JUNO will be a perfect observatory for nucleon decays. In this work I give an overview on the JUNO physics potential, the experimental status, and the performances of the JUNO detector for the different physics cases.

40th International Conference on High Energy physics - ICHEP2020

July 28 - August 6, 2020

Prague, Czech Republic (virtual meeting)

\footnotetext{
${ }^{*}$ Speaker
} 


\section{Introduction}

Reactor neutrino oscillation experiments provide a clean measurement of the oscillation parameters through the observation of the $\bar{v}_{e} \rightarrow \bar{v}_{e}$ transition. The typical short baseline makes the experiment insensitive to matter effects, and the fact that the goal is the observation of a disappearance measurement makes the transition insensitive to the $\delta_{c p}$ phase helping to obtain a clean measurement of the searched for parameters.

The oscillation regime is different according to the distance between the detector and the reactor as can be seen in Fig. 1(a), and JUNO is placed at the minimum of oscillation driven by the $\Delta m_{21}^{2}$ mass splitting. However it is sensitive to the interference of the different mass splittings and this feature makes JUNO able to discriminate between the two neutrino mass hierarchies $(\mathrm{MH})$ which is indeed the primary experimental goal.

The observation of anti-neutrinos is done through the inverse beta decay (IBD) process (i.e. $\left.\bar{v}_{e}+p \rightarrow e^{+}+n\right)$ allowing to perform a twofold coincidence between the prompt positron signal and the delayed neutron capture one for a clean signal selection (see Fig. 1(b)).

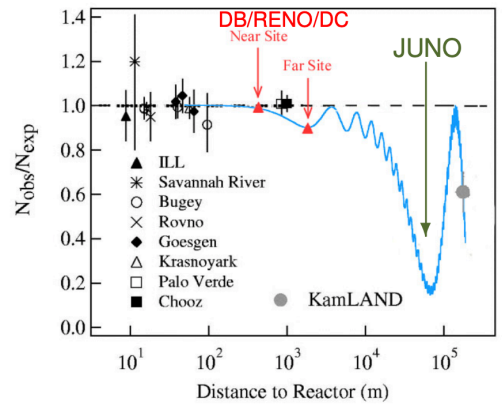

(a)

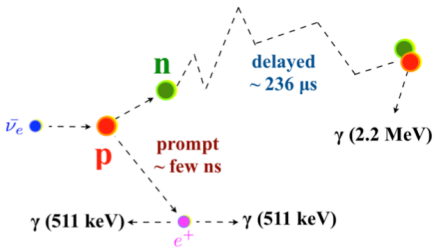

(b)

Figure 1: Ratio of observed events on expected events in case of no oscillation as a function of the distance between the reactor and the detector $(a)$, and cartoon showing the anti neutrino detection in JUNO $(b)$.

\section{JUNO experiment}

JUNO [1] is a medium-baseline reactor neutrino experiment. The baseline of $53 \mathrm{~km}$ from the Yangjiang and Taishan reactor complex, having a thermal power respectively of $17.4 \mathrm{GW}$ and $9.2 \mathrm{GW}$, was optimized in order to maximise the sensitivity to neutrino mass ordering.

The detector is based on liquid scintillator technology and JUNO will be the largest liquid scintillator detector ever built with its mass of 20 kton. The energy resolution is critical to perform a mass hierarchy measurement and the goal is to achieve $3 \%$ at $1 \mathrm{MeV}$, which means that about 1200 photoelectrons (p.e.) should be collected. To meet the requirements a large photo coverage is needed and indeed 17600 20-inch PMTs and 25600 3-inch PMTs will be installed in the JUNO detector. as shown in Fig. 2.

The detector is completed by a 35 kton water cherenkov detector (WCD) instrumented with 2400 20-inch PMTs which will be used as veto to tag muons passing close to the central detector, and 


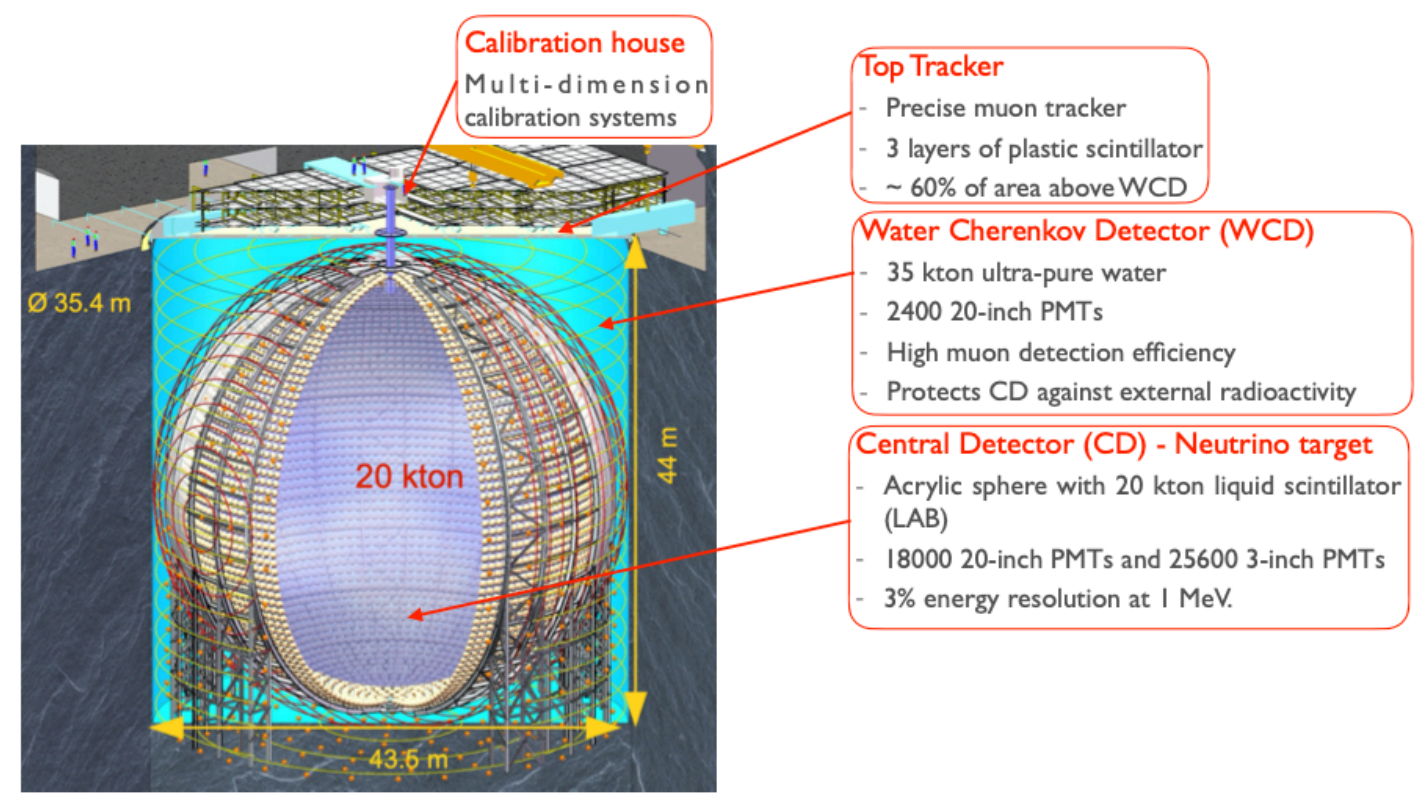

Figure 2: Schematic view of the JUNO detector.

the top tracker (TT) made of plastic scintillator strips which will allow to track muons above the detector for a better track reconstruction. The schematic view of the JUNO detector is shown Fig. 2.

\section{Physics reach of JUNO}

JUNO is a multipurpose neutrino observatory and it has a rich physics program in neutrino physics and astrophysics which goes beyond the mass hierarchy determination [2].

\subsection{Mass hierarchy determination}

As mentioned before, the interference term in the neutrino oscillation probability depends on the mass hierarchy i.e. on the sign of $\Delta m_{31}^{2}$ mas splitting, as can be seen in Fig. 3. Assuming an energy resolution meeting the requirements of $3 \%$ at $1 \mathrm{MeV}$, JUNO can perform a relative measurement (no constraint on $\Delta m_{31}^{2}$ ) at $\Delta \chi^{2}>9$ or an absolute measurement at $\Delta \chi^{2}>16$ accounting for constraints from long baseline experiments [1] .

In order to perform such a measurement the energy resolution (photo-electron statistics) is a critical parameter and the goal is to achieve $3 \%$ at $1 \mathrm{MeV}$. This implies a high scintillator light yield, a large PMT coverage and a comprehensive calibration strategy which will also assure an energy scale uncertainty at the sub-percent precision. An other important ingredient is a good knowledge of the reference energy spectrum with high energy resolution, which will be provided by the JUNO Taishan Antineutrino Observatory (TAO) [3], a ton-level, high energy resolution LS detector at 30 meters from one of the Taishan reactor cores.

The TAO detector will provide indeed a model independent reactor antineutrino spectrum which will be the reference for JUNO. In addition it will be a benchmark for investigation of the nuclear database, reactor monitoring and safeguard, and search for sterile neutrino. 


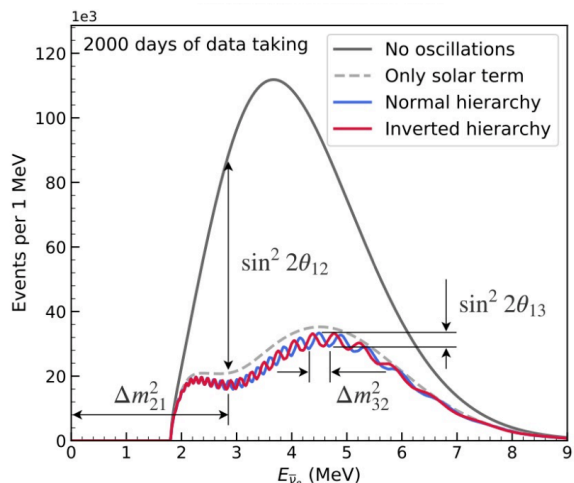

(a)

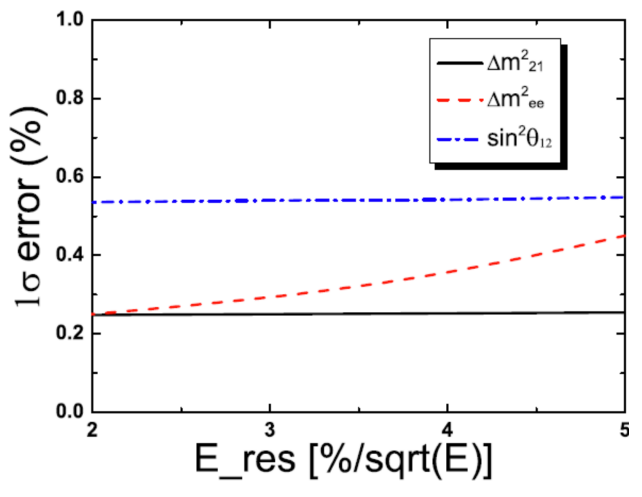

(b)

Figure 3: (a) Expected number of events as a function of anti neutrino energy in case of normal (blue) and inverted (red) mass hierarchy. (b) Precision on solar parameters as a function of the JUNO energy resolution.

\subsection{Precision measurements}

The second main goal of the JUNO experiment is the precise measurement with sub-percent precision, of the solar parameters $\sin ^{2} \theta_{12}$ and $\Delta m_{21}^{2}$. Such a measurement is essential to test consistency of neutrino oscillation framework. The current precision on these two parameters is at the level of $4.5 \%$ and $2.4 \%$ respectively [4] and the goal of JUNO is to achieve a precision at the level of $0.67 \%$ and $0.59 \%$ respectively.

It is important to notice that if the energy resolution is critical for the mass hierarchy measurement, it has no sizeable impact on the solar parameters measurements since their resolution is totally unaffected going from 3\% to 5\% energy resolution at $1 \mathrm{MeV}$ as shown in Fig. 3(b).

\subsection{Solar neutrinos}

Exploiting the detection of ${ }^{8} \mathrm{~B}$ neutrinos an independent measurement of $\Delta m_{21}^{2}$ can be performed with the same detector possibly shading new light on current tension between solar and reactor neutrinos measurement ( [5] and [6]).

Such a measurement is challenging because the low overburden results in a large contamination from cosmogenic isotopes requiring new veto strategies. In addition, given the detection via neutrinoelastic scattering, higher requirements in terms of radiopurity are needed: a threshold at $2 \mathrm{MeV}$ can be achieved with a ${ }^{238} \mathrm{U}$ and ${ }^{232} \mathrm{Th}$ radioactivity at the level of $10^{-17} \mathrm{~g} / \mathrm{g}$. If the requirements are met, with 10 years of data taking, about 60000 signal and 30000 background events are expected [7].

\subsection{Supernova neutrinos}

Neutrinos from supernovae are important messengers to understand the evolution of our Universe and JUNO will be able to detect neutrino from different sources.

Diffuse Supernova Neutrino Background (DSNB) gives an integrated neutrino flux from all past core-collapse events. A detection sensitivity at $3 \sigma$ after ten years can be achieved and in case no 


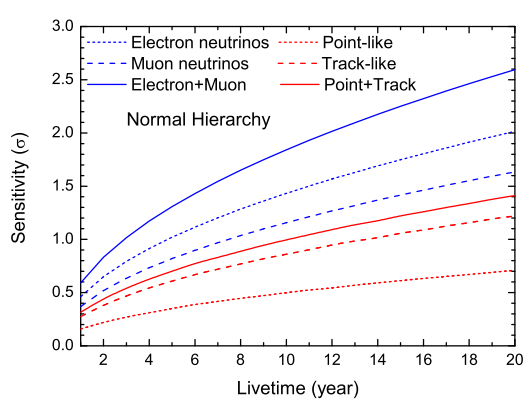

(a)

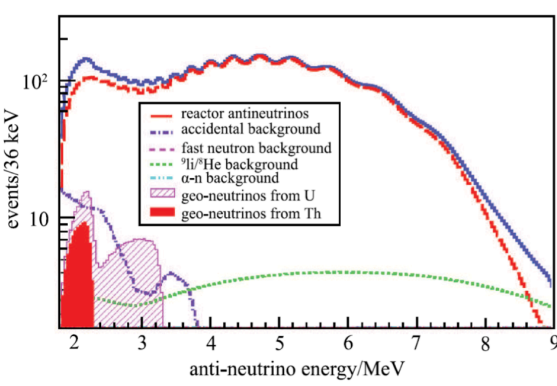

(b)

Figure 4: (a) Expected sensitivity on mass hierarchy from atmospheric neutrino analysis. (b) Expected geo-neutrino spectrum for 10 years of data taking.

signal is observed the best upper limit on the flux will be set: $0.2 \mathrm{~cm}^{-2} \mathrm{~s}^{-1}$ above $17.3 \mathrm{MeV}$ after 10 years.

For galactic core-collapse supernova neutrinos (CCSN) the flavour, energy spectrum and time evolution can be measured. JUNO has a low threshold at $0.2 \mathrm{MeV}$ and could observe about $5000 \mathrm{IBD}$ events due to $\bar{v}_{e}$ for a supernova explosion at $10 \mathrm{kpc}$, whereas the other neutrino flavours could be detected via $v-p$ elastic scattering.

Furthermore, if the threshold can be lowered to the challenging value of $10 \mathrm{keV}$, multi-messager astrophysics can be performed with a realtime monitoring of the $\mathrm{MeV}$ transient neutrino sky.

\subsection{Atmospheric neutrinos}

The observation of atmospheric neutrinos could provide an additional and independent measurement of the mass hierarchy exploiting matter effects. The sensitivity in terms of exposure can be seen in Fig. 4(a).

In addition the atmospheric neutrino measurement is sensitive to $\theta_{23}$ : the mixing angle $\theta_{23}$ could be measured with a precision of $6^{\circ}$ and the wrong octant can be ruled out at $1.8 \sigma$ and $0.9 \sigma$ in case of normal or inverted mass hierarchy respectively.

\subsection{Geo-neutrinos}

The observation of geo-neutrinos could provide important information to explore the origin and the thermal evolution of the Earth. Despite the IBD threshold at $1.8 \mathrm{MeV}$ and the fact that the anti-neutrino from reactors are dominant in the interest region below $3 \mathrm{MeV}$, an excess can be observed as shown in Fig. 4(b). Indeed the expected number of detected geo-neutrinos is about 500 per year and a precision of $6 \%$ on their energy spectra can be obtained in 10 years of data taking.

\subsection{Proton decay}

Proton decay observation would be a fundamental discovery casting light on possible theories beyond the Standard Model.

JUNO would provide a competitive sensitivity for the channel $p \rightarrow \bar{v}+K^{+}$thanks to the clear 


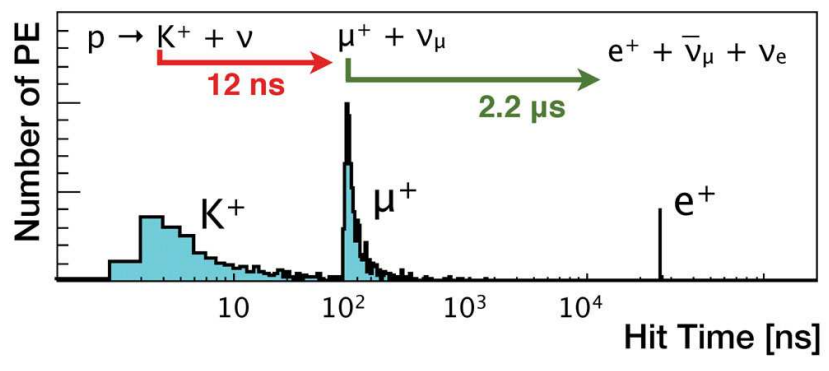

(a)

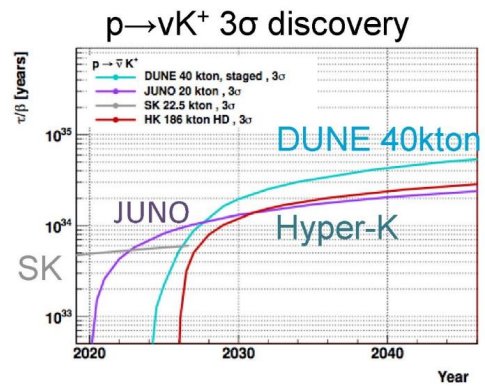

(b)

Figure 5: (a) Cartoon showing the proton decay chain with the reconstructed signal in time. (b) JUNO sensitivity to $p \rightarrow \bar{v}+K^{+}$decay versus time and sensitivity for DUNE [8] and Hyper-Kamiokande [9].

identification of three signals in a specific time coincidence. Indeed the kaon would decay with a lifetime $(\tau)$ of $12 \mathrm{~ns}$ into muon which in turn will decay with a $\tau$ of $2.2 \mu$ s into positron as shown in Fig. 5(a).

After 10 years of data taking a sensitivity of $2 \times 10^{34}$ years can be achieved as shown in Fig. 5(b).

\section{Conclusions}

JUNO will be the largest liquid scintillator detector ever built and will start data taking in 2022 with the main goal of determining the neutrino mass hierarchy and measuring with subpercent precision the solar parameters $\theta_{12}$ and $\Delta m_{21}^{2}$. The rich program in neutrino physics and astrophysics of JUNO goes far beyond the main objectives: JUNO is indeed a multipurpose neutrino observatory which will detect solar, atmospheric and geo-neutrinos, search for supernova neutrinos, and for proton decay.

\section{References}

[1] Z. Djurcic et al. [JUNO], [arXiv:1508.07166 [physics.ins-det]].

[2] F. An et al. [JUNO], J. Phys. G 43, no.3, 030401 (2016).

[3] A. Abusleme et al. [JUNO], [arXiv:2005.08745 [physics.ins-det]].

[4] P.A. Zyla et al. [Particle Data Group], Prog. Theor. Exp. Phys. 2020, 083 C01 (2020).

[5] K. Abe et al. Phys. Rev. D, 94(5):052010, 2016.

[6] A. Gando et al. Phys. Rev. D, 88(3):033001, 2013.

[7] A. Abusleme et al. [JUNO], [arXiv:2006.11760 [hep-ex]].

[8] R. Acciarri et al. [DUNE] [arXiv:1512.06148 [hep-ex]].

[9] K. Abe et al. [Hyper-Kamiokande] [arXiv:1109.3262 [hep-ex]]. 